\title{
Event-Related Phase-Amplitude Coupling: a comparative study
}

\author{
A. Bachiller, J. Gomez-Pilar, J. Poza, P. Núñez, C. Gómez, A. Lubeiro, V. Molina, R. Hornero
}

\begin{abstract}
The aim of this study was to explore the coupling among neural oscillations in different frequency bands using two approaches: conventional phase-amplitude coupling (PAC) and a novel event-related PAC. Both measures were applied to the electroencephalographic activity from 20 healthy volunteers. The results showed that the phase of alpha band modulated gamma power. Event-related PAC measures the coupling between frequency rhythms without losing of temporal resolution. Therefore, it may provide further insights into the characterization of brain dynamics.
\end{abstract}

\section{INTRODUCTION}

$\mathrm{N}$ EURAL oscillations are the main mechanism for enabling coordinated activity during normal brain functioning. The rhythms in different frequency bands can interact among each other in behaviorally meaningful ways [1]. This phenomenon is known as 'Cross-Frequency Coupling' (CFC). CFC is postulated as a key mechanism for information exchange and the integration of distinct processes mediated by distinct brain rhythms [2].

Different types of CFC have been identified; oscillations at different frequencies could be coupled in several ways involving either the phase, amplitude or the frequency of the signals [3]. In this regard, phase-to-amplitude coupling (PAC) is the most widely used. PAC describes the statistical dependence between the phase of a low-frequency brain rhythm and the amplitude of a high-frequency component of brain activity [1]. The factors that contribute to these mechanisms are still not completely understood. Nevertheless, it has been proposed that the phases of lowfrequency oscillations reflect fluctuations of membrane potentials in a brain area that affects the amplitude of highfrequency oscillations in that region [4].

Conventionally, PAC is estimated over a long fixed time window with a lost of temporal resolution [5]. In addition, several techniques such as the concatenation of time segments across trials can introduce spurious PAC due to

This research was supported in part by the projects TEC2014-53196-R of 'Ministerio de Economía y Competitividad' (MINECO) and FEDER, the project VA059U13 of 'Consejería de Educación', the project FIS PI1 102303 of 'Instituto de Salud Carlos III and the project GRS 932/A/14 of 'Gerencia Regional de Salud de Castilla y León'. A. Bachiller and J. Gomez-Pilar were in receipt of a PIF-UVA grant from the University of Valladolid, P. Núñez was in receipt of a 'Promoción de empleo joven e implantación de la Garantía Juvenil en I+D+i' grant from MINECO and the University of Valladolid.

A. Bachiller, J. Gomez-Pilar, J. Poza, P. Núñez C. Gómez and R. Hornero are with the Biomedical Engineering Group, University of Valladolid, Spain.

A. Lubeiro and V. Molina are with the Faculty of Medicine, University of Valladolid, Avenida Ramón y Cajal 7, 47007 Valladolid. edge artifacts [5]. Nevertheless, a novel approach allows measuring transient PAC in an 'event-related' way [2], [5]. The aim of this study was to validate the novel event-related PAC (ERPAC) approach by comparing it with conventional PAC and, therefore, obtaining a transient measure of PAC time dynamics.

\section{MATERIALS AND METHODS}

\section{A. Subjects}

Twenty healthy volunteers were recruited for the study (age 29.32 \pm 9.80 years, mean \pm standard deviation). Written informed consent was obtained from all healthy volunteers.

\section{B. Electroencephalographic Recordings}

Electroencephalographic (EEG) recordings were performed while subjects underwent a 3-stimulus auditoryoddball task. Participants heard a random series of 600 binaural tones consisting on target $(500 \mathrm{~Hz})$, distractor $(1000$ $\mathrm{Hz})$, and standard $(2000 \mathrm{~Hz})$, with probabilities of 0.20 , 0.20 , and 0.60 respectively [6].

For each subject, $13 \mathrm{~min}$ of auditory response EEG activity were acquired at a sampling rate of $500 \mathrm{~Hz}$ with an EEG system (Brain Products GmbH; Munich, Germany). Data recording consists of 32 sensors placed according to the International 10/20 System. Channels TP9, TP10, PO9 and PO10 were discarded due to abundant muscle artifacts throughout. Filtering and artifact-rejection procedures were carried out following the steps described in [6]. Finally, CFC analysis was carried out over P3 electrode, since parietal area has been widely associated with memory and cognitive processes [4].

\section{PAC vs. ERPAC}

PAC has been proposed as a mechanism to coordinate the timing of neuronal firing within local neural networks [4]. Conventional PAC algorithms obtain an averaged PAC value across a defined time window, which is bounded by the frequency of the coupling phase. In particular, the time window should be, at least, one full cycle of the lowfrequency signal; hence it involves a loss of temporal resolution [5]. By contrast, ERPAC approach attempts to solve the loss of temporal resolution by performing PAC estimation at each time point, across-trials [2], [5].

\section{CFC estimation}

Both PAC and ERPAC were estimated at P3 electrode using the 'phase-locking value' (PLV) method (for more details, please see [2], [7], [8]) .

In this study, we calculated PAC under a 500 ms-length 
temporal window (from 100 to $600 \mathrm{~ms}$ after stimulus onset). On the other hand, ERPAC was estimated across trials using a temporal window of $20 \mathrm{~ms}$ [2], which gives a time-variant measure of PAC. Finally, ERPAC values were averaged over the [100 600] ms temporal window (Fig. 1b), in order to compare both measures.

\section{E. Statistical analysis}

We used surrogate data test to determine whether CFC was greater than what would be observed by chance [3], [8]. Two different surrogate procedures were used in this study. We generated surrogate data through a random permutation of phases (commonly know as 'phase shuffling' [3]) for PAC approach. In the case of ERPAC, we carried out a resampling analysis that randomized the inter-trial relationship between amplitude and phase [5]. In both cases, the change from surrogate data was calculated using a $z$ score measure [5].

\section{RESULTS AND DISCUSSION}

PAC and ERPAC analyses were computed over a sweep of frequencies: (i) from $4 \mathrm{~Hz}$ to $13 \mathrm{~Hz}$, with a step of $1 \mathrm{~Hz}$ and a bandwidth of $2 \mathrm{~Hz}$ for low frequencies; and (ii) for high frequencies from $30 \mathrm{~Hz}$ to $110 \mathrm{~Hz}$, with a step of $10 \mathrm{~Hz}$ and a bandwidth of $30 \mathrm{~Hz}$. After surrogate analysis, we obtained a comodulogram for each subject. This graph depicts the CFC, measured by $z$-score values, for each pair of low- and high-frequency values. Fig. 1a showed the conventional PAC patterns computed over a post-stimulus [100 600] ms time window. To provide a meaningful comparison between both methods, we averaged ERPAC values over the same post-stimulus [100 600] $\mathrm{ms}$ time window (Fig. 1b).

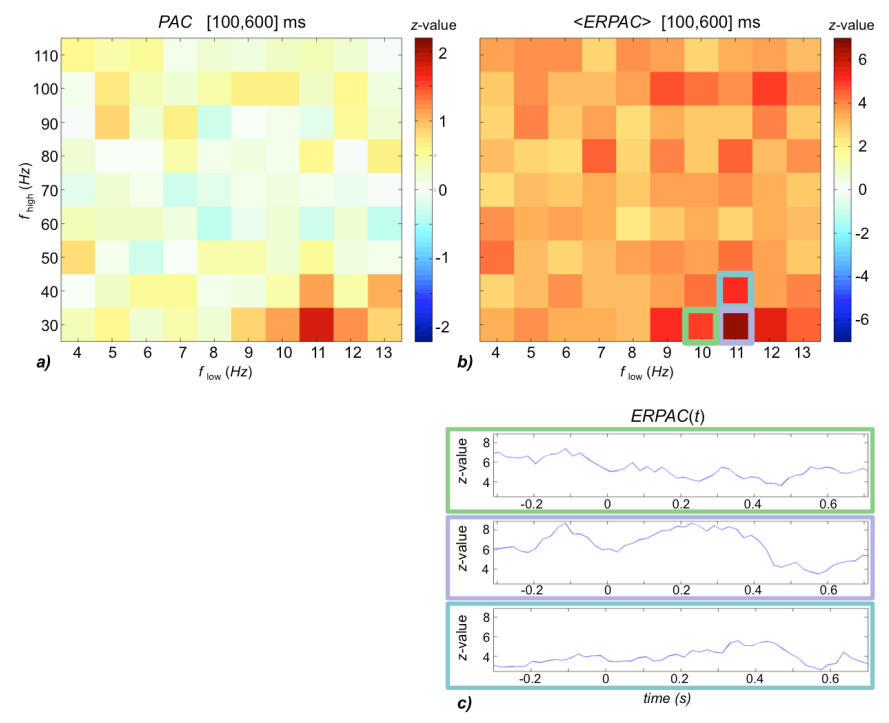

Fig. 1. Averaged CFC patterns over all healthy volunteers at P3 electrode. (a) Phase-amplitude comodulogram of surrogated conventional PAC. (b) Averaged comodulogram over post-stimulus [100 600] ms-time window of surrogated ERPAC. (c) Surrogated ERPAC time-variation for the three pair of frequencies highlighted in (b).
In agreement with previous results, both approaches displayed a meaningful coupling between the phases at alpha band (approximately in the range [8 13$] \mathrm{Hz}$ ) and the amplitudes at beta and low-gamma band (frequencies in the range [15 45] Hz). The phase of alpha oscillations may coordinate neuronal processing reflected in gamma power in humans [9], [10]. These findings could evidence the role of alpha rhythms as a core feature of cortical communication and cognition [9] and it may support the idea that alpha activity is associated with functional inhibition process [10].

How the neuronal dynamics in the alpha and gamma bands interact remains unknown. In this regard, our findings confirm the validity of ERPAC approach and provides complementary information about the time-evolution of coupling patterns (Fig. 1c) [5]. Further researches can provide novel insights into how neural networks process and share information. In addition, future work will assess the relation between ERPAC findings and cognitive impairments in neuropsychiatric disorders, such as schizophrenia.

\section{CONCLUSION}

ERPAC is a meaningful measure of dynamic CFC. It can reveal novel insights into the characterization of integration mechanisms and distributed neural information processing. Current and future CFC studies are also motivated by the hypothesis that patterns of CFC could be altered in neuropsychiatric disorders.

\section{REFERENCES}

[1] R. T. Canolty and R. T. Knight, "The functional role of crossfrequency coupling," Trends Cogn. Sci., vol. 14, no. 11, pp. 506$515,2010$.

[2] S. I. Dimitriadis, N. A. Laskaris, M. P. Bitzidou, I. Tarnanas, and M. N. Tsolaki, "A novel biomarker of amnestic MCI based on dynamic cross-frequency coupling patterns during cognitive brain responses," Front. Neurosci., vol. 9, no. OCT, pp. 1-17, 2015 V. Jirsa and V. Müller, "Cross-frequency coupling in real and virtual brain networks," Front. Comput. Neurosci., vol. 7, Jul. 2013.

[4] J. Fell and N. Axmacher, "The role of phase synchronization in memory processes.," Nat. Rev. Neurosci., vol. 12, no. 2, pp. 105118, 2011.

[5] B. Voytek, M. D’Esposito, N. Crone, and R. T. Knight, "A method for event-related phase/amplitude coupling," Neuroimage, vol. 64, no. 1, pp. 416-424, 2013.

[6] A. Bachiller, J. Poza, C. Gómez, V. Molina, V. Suazo, and R. Hornero, "A comparative study of event-related coupling patterns during an auditory oddball task in schizophrenia.," J. Neural Eng., vol. 12, no. 1, p. 016007, 2015.

[7] B. Voytek, R. T. Canolty, A. Shestyuk, N. E. Crone, J. Parvizi, and R. T. Knight, "Shifts in gamma phase-amplitude coupling frequency from theta to alpha over posterior cortex during visual tasks," Front. Hum. Neurosci., vol. 4, no. October, p. 191, 2010.

[8] B. L. Foster and J. Parvizi, "Resting oscillations and crossfrequency coupling in the human posteromedial cortex," Neuroimage, vol. 60, no. 1, pp. 384-391, 2012.

[9] M. Bonnefond and O. Jensen, "Gamma Activity Coupled to Alpha Phase as a Mechanism for Top-Down Controlled Gating," PLoS One, vol. 10, no. 6, p. e0128667, 2015.

[10] J. I. Berman, S. Liu, L. Bloy, L. Blaskey, T. P. L. Roberts, and J. C. Edgar, "Alpha-to-gamma phase-amplitude coupling methods and application to autism spectrum disorder.," Brain Connect., vol. 5, no. 2, pp. 80-90, 2015. 